\title{
TRANSFORMACIONES NECESARIAS Y CÓMO CONSTRUIRLAS PARA CONTRIBUIR A ELEVAR EL NIVEL DE DESARROLLO HUMANO EN LA REGIÓN DESDE LA GERENCIA DE SISTEMAS DE SALUD
}

Recibido: marzo del 2018

Aceptado: agosto del 2018

Juan José Ospina Ramírez ${ }^{1}$, Ximena Mejía Marín²

Ensayo de reflexión

\section{Resumen}

La OMS define la calidad como "el grado en el que los servicios de salud prestados a personas y poblaciones aumentan la probabilidad de lograr los resultados sanitarios deseados y son coherentes con los conocimientos profesionales del momento" (1). Así, se entiende la calidad desde las normas ISO 9000 como el grado en el que un conjunto de características inherentes cumple con la necesidad o expectativa establecida, generalmente implícita y obligatoria $(2,3)$. El concepto de calidad en la atención médica más utilizado es el que definió Avedis Donabedian: "el tipo de atención que maximiza el bienestar del paciente, después de tener en cuenta el balance de pérdidas y ganancias esperadas, contemplando el proceso de atención en todas sus partes" (4). Se pretende, entonces, relacionar de manera reflexiva, cuáles han sido los avances de la normativa colombiana a lo largo de los últimos 25 años, para incentivar las trasformaciones necesarias y cómo debemos, en conjunto, el colectivo de trabajadores del área de la salud, construir para contribuir a elevar el nivel de desarrollo humano en la región desde la gerencia de sistemas de salud. El texto quiere invitar al lector a hacer una aproximación de manera general al SOCG (Sistema Obligatorio de Garantía de la Calidad) desde la gerencia de sistemas de salud y evidenciar cómo ha sido su evolución a lo largo de los últimos 25 años, así como plantear estrategias administrativas y gerenciales de marketing, las cuales se evidencian como fallos del mercado con desarrollos inapropiados y cómo el gobierno colombiano, a través de la Resolución 429 de 2016 (5), plantea hasta ahora un paradigma con necesidades prontas de implementación.

Palabras clave: articulación y modulación, formación de redes, mercados de asistencia sanitaria, producción sanitaria, salud, sanidad pública.

${ }^{1}$ Médico, epidemiólogo clínico, gerente de calidad y auditor en salud, magíster en Gerencia de Sistemas en Salud, Fellow MBA de Alta Gerencia, doctorante en Ciencias de la Educación. Docente de la Universidad Tecnológica de Pereira, área maternoinfantil.

2 Enfermera profesional, especialista en Auditoria en Salud y magíster en Gerencia de Sistemas en Salud. Secretaria de Salud Municipal en Pereira y docente universitaria de Gestión de Docencia y Servicio de la Universidad Cooperativa de Colombia. 


\title{
NECESSARY TRANSFORMATIONS AND HOW TO BUILD THEM FROM HEALTH SYSTEM MANAGEMENT IN ORDER TO CONTRIBUTE TO ELEVATE LEVEL OF HUMAN DEVELOPMENT IN THE REGION
}

\author{
Juan José Ospina Ramírez ${ }^{1}$, Ximena Mejía Marín²
}

\section{Reflection essay}

\section{Abstract}

The World Health Organization defines quality as "the extent to which health care services provided to individuals and patient populations improve desired health outcomes" (1). That way, it is seen through the ISO 9000 regulations as the rate in which a set of attached characteristics meets an established need or expectation, one that would be implicit and mandatory ( 2 , 3 ). The most used concept of quality in medical attention is the one defined by Avedis Donabedian: "it's the kind of attention that maximizes patients' well-being, once the balance of gains and losses is taken into account, so the process can be observed from all possible dimensions" (4). Thus, our objective is to describe and reflect about the progress of Colombian legislation during the last 25 years, in order to boost the necessary transformations and the way we must, as a whole team of workers of the health area, contribute to elevate the level of human development in the region from health area systems. The text invites the reader to approach from the management of health systems the Mandatory Quality Assurance System. That way, it is possible to prove its evolutive process along the last 25 years and plan marketing strategies and show how the Colombian government, through the Resolution 429 of 2016 (5) poses a new paradigm with urgent needs of implementation, given that previous strategies are presented as failures in the market, with inappropriate developments. 


\title{
TRANSFORMAÇÕES NECESSÁRIAS E COMO CONSTRUII-LAS PARA CONTRIBUIR A AUMENTAR 0 NÍVEL DE DESENVOLVIMENTO HUMANO NA REGIÃO A PARTIR DA GERÊNCIA DE SISTEMAS DE SAÚDE
}

\author{
Juan José Ospina Ramírez ${ }^{1}$, Ximena Mejía Marín²
}

\section{Ensaio de reflexão}

\section{Resumo}

A Organização Mundial da Saúde define a qualidade como "o grau no qual os serviços de saúde prestados a pessoas e populações aumentam a probabilidade de atingir os resultados sanitários desejados e são coerentes com os conhecimentos profissionais do momento" (1). Assim, entende-se a qualidade sob as normas ISO 9000 como o grau em que um conjunto de características inerentes cumpre com a necessidade ou expectativa estabelecida, geralmente implícita e obrigatória $(2,3)$. O conceito de qualidade no atendimento médico mais utilizado é o que definiu Avedis Donabedian: "o tipo de atendimento que maximiza o bem-estar do paciente, depois de considerar o balanço de perdas e ganhos esperados, contemplando o processo de atenção em todas as suas partes" (4). Portanto, pretende-se relacionar, de maneira reflexiva, quais têm sido os avanços da normativa colombiana ao longo dos últimos 25 anos, para incentivar as transformações necessárias e como, em conjunto, o coletivo de trabalhadores da área da saúde deve construí-las para contribuir a aumentar o nível de desenvolvimento humano na região a partir da gerência de sistemas de saúde. Este texto pretende convidar o leitor a se aproximar, de maneira geral, do Sistema Obrigatório de Garantia da Qualidade a partir da gerência de sistemas de saúde e evidenciar como foi sua evolução nos últimos 25 anos, bem como propor estratégias administrativas e gerenciais de marketing, as quais são identificadas como falhas do mercado com desenvolvimento inapropriado, e como o governo colombiano, por meio da Resolução 429 de 2016 (5), apresenta, até o momento, um paradigma com necessidades urgentes de implantação. 
Luego de la postura por Avedis Donabedian se propuso que se abordara la calidad de la atención en salud desde la atención médica individual, a través de dos aspectos: el primero mediante la aplicación del conocimiento médico y de la tecnología, con el fin de obtener el máximo de los beneficios con un mínimo de riesgos, de acuerdo a las preferencias de cada usuario. El segundo aspecto se refiere al manejo de la relación personal con el usuario que debe ser acorde a los principios éticos, a los convenios sociales y a las expectativas y necesidades legítimas del usuario.

Además, se definieron siete pilares de la calidad: eficacia, efectividad, eficiencia, optimización, aceptabilidad, legitimidad y equidad (6), pero se debe hacer una referencia fuerte a que los atributos de la calidad en la atención de salud siempre irán a estar medidos o regulados por accesibilidad, oportunidad, seguridad, pertinencia, continuidad y satisfacción del usuario (7). Para analizar la problemática de la mala calidad o no calidad en la atención de salud es necesario mencionar una de sus consecuencias y es que la falta de calidad hace al servicio de salud inseguro, lo que ocasiona daño material o humano, causa desconfianza $y$ acciones legales que generan reacciones prácticas de medicina defensiva (8).

El gobierno colombiano, a través de la Resolución 429 de 2016 (5), promulgó la Política de Atención Integral en Salud (PAIS), la cual pretende generar mejores condiciones de salud para la población a través de la regulación de la intervención sectorial e intersectorial, sustenta-

do en lo dispuesto por un gran volumen de normatividad previa que comprende desde la Ley 100 de 1993 y sus posteriores modificaciones, incluyendo la Ley 1438 de 2011 (9), la cual pretendía fortalecer la atención primaria en salud, el plan decenal de salud pública y hasta la de más reciente expedición, la Ley Estatutaria 1751 de 2015 (10), que luego de una gran controversia consagró por fin la salud como un derecho fundamental.

En Colombia se ha realizado un desarrolo bastante importante en materia legislativa, pero esta no ha podido resolver los graves problemas por los que atraviesa el sector y que han sido reconocidos por el propio gobierno: fragmentación y desintegración de la atención; baja resolutividad (11), baja eficiencia donde se procurase por la mejor utilización social y económica de los recursos, servicios y tecnologías disponibles para garantizar el derecho a la salud de toda la población (10). Se documenta una alta carga de enfermedad llevando, a la alta complejidad, niveles de atención III y IV con la carga de siniestralidad en salud y siniestralidad financiera. Fallos del mercado con desarrollos inapropiados de marketing, entendiendo que el marketing es ajeno a la planeación y a las políticas de la salud. El Decreto 1011 de 2006 define el SOGC (Sistema Obligatorio de Garantía de Calidad en Salud) de atención en salud como el conjunto de instituciones, normas, requisitos, mecanismos y procesos liberados y sistemáticos que desarrolla el sector salud para generar, mantener y mejorar la calidad de los servicios de salud en el país (12). Las instituciones y profesionales de salud y de otras áreas deben tomar nota de todas las posibilidades, los aciertos y las limitaciones que ofrecen estos sistemas de mercadeo (13). 
Así mismo, el artículo 3 del Decreto 1011 de 2006 establece que las acciones que desarrolle se orientaran a la mejora de los resultados de la atención en salud, siempre centrados en el usuario, más allá de la verificación de existencia de estructura o de la documentación de procesos, los cuales solo constituyen los prerrequisitos para alcanzar los mencionados resultados y señala que se debe cumplir estas características:

1. Accesibilidad

2. Oportunidad

3. Seguridad

4. Pertinencia

5. Continuidad

Asimismo, hacen parte como componentes del SOGC:

\section{Sistema único de habilitación}

2. Auditoría para el mejoramiento de la calidad (Pamec)

3. Sistema único de acreditación

4. Sistema de información para la calidad

Diversos sistemas de salud en el mundo están enfrentando el gran reto de las enfermedades crónicas no trasmisibles (6); no lejos de estas problemáticas se encuentra nuestro departamento de Risaralda, donde se refleja la misma problemática (14), por lo que se haría más evidente la utilidad de una herramienta que permita el geomarketing, enmarcado en un simulador demográfico para ubicar a todas las personas que tienen algunas de estas patologías prevalentes, toda vez que permitiría diseñar estrategias de marketing uno a uno, que lleven a modificar conductas, costumbres y hábitos malsanos como el sedentarismo, los malos hábitos alimenticios, el alcoholismo, etc., modificados estos luego de una intervención oportuna por hábitos que lleven a prolongar los estados de bienestar y salud de todas las personas de una comunidad, ciudad o nación; también nos encontramos con padecimientos como el no reconocimiento de incentivos al talento humano en salud (15).

Al comprender esto a nivel mundial, desafortunadamente como programas encaminados a un ofrecimiento de recompensas de carácter económico, dejan atrás los incentivos que denotan un esfuerzo menor para las organizaciones como aquellos no financieros y que a lo largo son más reconocidos por el talento humano.

Además de comprender el interior de las organizaciones, el trabajador debe sentirse valorado y cuidado por la organización que lo contrata, mediante acciones incentivadoras tales como:

- Reconocimiento de logros

- Oportunidades de promoción laboral

- Proporcionar medios necesarios para trabajar con calidad y de forma autónoma "recursos"

- Fomentar el aporte de sus propias ideas y la participación en todos los ámbitos, incorporando al propio trabajador en la ruta de acreditación institucional (15).

Además de una clara falla regulatoria entre EAPB e IPS, lo cual podría controlarse con una estrategia de marketing de calidad tecnológica enlazada con una es- 
trategia de imagen y de precio, algo muy semejante a mantener un observatorio local en cada departamento que permita saber cuál es el comportamiento del mercado en salud, así pues, se le podría dar un control a estos incentivos negativos, lo que permitiría realizar una adecuada balanza al controlar la inequidad y la corrupción; el maltrato al usuario, las percepciones de atención en salud de manera negativa, el desorden administrativo, entendiéndose en el imaginario corporativo y administrativo al interior de las organizaciones hospitalarias como riesgo corporativo-empresarial, toda vez que aumenta el riesgo en salud, cuando la responsabilidad recae sobre los gobernantes, siendo los alcaldes municipales y sus respectivos secretarios de salud los presidentes de las juntas directivas.

Será entonces que se requiere mayor gobierno al implementar un mecanismo de aumento de la calidad, lo que permitirá una optimización en la gobernanza local (16), donde se haga a un lado a los modelos de prestación de servicios de salud, morbicéntrico y centrado en los actores, y se encuentren mecanismos de humanización en salud (9).

Al tener en cuenta todo lo anteriormente expresado, se observa que para la prestación de estos servicios de salud se deben cumplir unos requisitos exigidos por la norma, se pensaría entonces que todas las organizaciones en el marco de la implementación de estas políticas deben contar con una persona cuyo perfil profesional le permita un seguimiento, no solo a la Resolución 743 de 2013 y

38 Resolución 2003 de 2014, además, que permita un sistema de evaluación y ca- lificación de actores: "Ranking de Satisfacción EPS 2017 Oficina de Calidad”14 y habilite también un ranking de IPS por medio del Mocas (17).

Desde este análisis, el asunto está basado en el argumento del porqué las territoriales de salud necesitan una trasformación e incrementar las capacidades de gobierno. Desde la gobernanza en el sistema territorial podríamos hablar de una reforma a la Ley 715 de 2001, modificada por la Ley 863 de 2003, "Por la cual se establecen normas tributarias, aduaneras, fiscales y de control para estimular el crecimiento económico y el saneamiento de las finanzas públicas", donde la corte constitucional se declaró inhibida de fallar sobre el artículo 49 de la Ley 863 de 2003, mediante Sentencia C91004 de 21 de septiembre de 2004, del magistrado ponente, doctor Rodrigo Escobar Gil.

En dicha ley se reglamenta una parte de los ingresos de los municipios de nuestro país, a saber, las transferencias a salud y educación, de propósito general fue diseñada como un nuevo instrumento de las finanzas. En razón de su cumplimiento, los municipios y los departamentos vieron un cambio en sus responsabilidades frente al manejo de la educación, la salud pública y el sistema hospitalario. Se trata de una ley de carácter orgánico porque sus normas poseen un nivel jerárquico superior a la Ley 100 de 1993, deroga y sustituye a la Ley 60 de 1993 y modifica el sistema de competencias y recursos generado por la Ley 10 de 1990. Es allí donde se establece como competencias departamentales adoptar, difundir, implantar, ejecutar y 
evaluar en el ámbito departamental las normas, políticas, estrategias, planes, programas y proyectos del sector salud y del sistema general de seguridad social en salud que formule y expida la nación o en armonía con estas $(6,18)$. Esta fue diseñada como un nuevo instrumento de las finanzas intergubernamentales en nuestro país.

En nuestro medio el mercado de la salud en el imaginario de transacción de compra y venta de servicios de salud, la operación cotidiana del sistema en el marco de la estandarización por una PAIS (Política de atención integral en salud), además de un MIAS (Modelo de Atención Integral en Salud) ahora MAITE (Modelo de Acción Integral Territorial, así quedó establecido al conocerse la resolución para observaciones que hizo pública el Ministerio de Salud y Protección social y que deroga integralmente las resoluciones 429 de 2016 (5) y 489 de 2019, toda lo anterior en el marco de la priorización desde las diferentes RIAS (Rutas Integrales de Atención en Salud). Entendiendo todo este libreto parte de los actores, pero que ocurre con el componente del territorio, ¿Que le compete y corresponde a nuestros gobernantes en el territorio?, teóricamente todos son responsables de la salud y son responsables de labores de salud pública, por lo menos no lo que está en función de la atención individual del enfermo si no con toda la gente sana, que tienen un papel en él, esperemos que el MAITE al ser fundamentado en la atención primaria en salud (APS), con enfoque de salud familiar y comunitaria, el cuidado, la gestión integral del riesgo y el enfoque diferencial para los distintos territorios y poblaciones, sea la nueva resolución especifica como hoja de ruta de las diferentes líneas de acción.

En ese sentido, el Modelo de Acción Integral Territorial (MAITE), es el nuevo marco operacional de la Política de Atención Integral en Salud (PAIS), y constituye el instrumento de gestión para mejorar las condiciones de salud de la población a través de acciones coordinadas entre los agentes del sistema de salud, agentes de otros sistemas y las comunidades, bajo el liderazgo del departamento o del distrito, que identifica prioridades y establece acciones que se operativizan a través de acuerdos interinstitucionales y comunitarios, con el acompañamiento y facilitación del Ministerio de Salud.

Seres humanos en diferentes mercados grave error en mi opinión, en el primer mercado de EPS de aseguramiento, se entiende gente como un afiliado o un cotizante asegurado para que se le realice la atención en una red de prestadores; en el segundo mercado EPS e IPS, mercado de transacciones de compra y venta de servicios de salud, tecnologías de salud, los cuales están dado por las atenciones del POS y el tercer mercado de la salud pública, el que se origina a partir de direcciones territoriales de salud (DTS) y prestadores de servicios de salud de atenciones colectivas (PPS. $\mathrm{AC}$ ), este último identificado por el plan de intervenciones colectivas (PIC), siguiendo las directrices de la Resolución 518 de 2015 del plan de salud pública de intervenciones colectivas, enmarcado en el artículo 4 que habla de la gestión de salud pública (21). 
En razón de su cumplimiento, los municipios y los departamentos vieron un cambio en sus responsabilidades frente al manejo de la educación, de la salud pública y del sistema hospitalario. Se trata entonces la Ley 715 de 2001, entendiéndola como de carácter orgánico, porque sus normas poseen un nivel jerárquico superior a la Ley 100 de 1993 y deroga y sustituye a la Ley 60 de 1993 y modifica el sistema de competencias y recursos generado por la Ley 10 de 1990, con sus fondos locales de salud.

La Ley 715 de 2001 de diciembre 21, por la cual se dictan normas orgánicas en materia de recursos y competencias de conformidad con los artículos 151, 288, 356 y 357 (acto legislativo 01 de 2001 de la Constitución Política) y se dictan otras disposiciones para organizar la prestación de los servicios de educación y salud, entre otros. Simplifica el flujo de recursos porque fusiona el situado fiscal modificado por el sistema general de participaciones municipales para inversión social en salud y diferencia los flujos por el objeto, distinguiendo en forma más precisa que va para la salud pública y que valores en subsidios son de oferta o de demanda. También modifica los criterios de competencia de la siguiente manera: los municipios manejan la demanda y los departamentos los subsidios de oferta (22), además de conformidad con lo señalado por el artículo 56 de la Ley 715 de 2001, las Entidades Departamentales y Distritales de Salud realizarán el proceso de inscripción en

40 el Registro Especial de Prestadores de Servicios de Salud (12).
Así, en el entendido de la Ley 715 de 2001, la cual asigna los recursos por resultados, ¿cuántos niños o adultos están matriculados? y ¿cuántos están afiliados al régimen subsidiado? Se distribuye de la siguiente manera: $58,5 \%$ de participación para educación, $24,5 \%$ de participación para salud, 5,4\% de participación para agua potable, $11,6 \%$ de propósito general (cultura, deportes, alimentación escolar entre otros).

Otro de los cambios implementados con la puesta en marcha de la Ley 715 de 2001 fue la del manejo de los recursos de la salud que reemplaza los fondos locales de salud, con la Ley 60/83 la tesorería de la nación giraba un cheque a los fondos comunes del municipio. Con este cambio, el gobierno espera evitar desviaciones, embargos y malos usos por parte de los municipios, es protagonista entones el marketing uno a uno con el desarrollo de la herramienta del geomarketing como parte de una estrategia corporativa de la gobernanza, al dividir la segmentación del mercado del departamento y de la mano con la investigación del mercado en el departamento de Risaralda, permitiéndose comprender el mercado para valorar el bienestar de la sociedad articulado con el marketing social, donde lo social, lo económico y lo ambiental permitan la medición de la calidad en salud $(19,20,23)$.

¿Qué puede hacer entonces en el departamento de Risaralda?, ¿modernizar los programas de salud pública presentando una automatización en ellos?, siendo los secretarios de salud y presidentes de las juntas directivas de la red hospitalaria del régimen subsidiado, será im- 
portante tener herramientas de gestión administrativa y regular los precios del mercado entre EPS e IPS, además de aumentar la capacidad de gobierno y la gobernanza de la calidad de atención de los servicios de salud del departamento de Risaralda.

$\mathrm{Al}$ conocer entonces cuáles son los indicadores de la calidad que contemplan las dimensiones de efectividad de la atención, de seguridad del paciente, de gestión del riesgo y de experiencia de la atención, para las IPS, EAPB y entidades territoriales de salud, lo que se complementa además con ¿cuáles son los indicadores de vigilancia y control que permiten medir la gestión de la calidad en la atención en salud del departamento de Risaralda?, comprendiendo el imaginario de nuestros usuarios como: ¿cuál es la percepción de los habitantes del departamento de Risaralda en cuanto a la calidad de la atención de los servicios de salud del departamento? Será entonces que la Resolución 0256 de 2016, que derogó la Circular Única 045 (24), logra identificar completamente cuáles son los indicadores que evalúan el monitoreo de la calidad o será más bien que a pesar de la rigurosidad enmarcada por dominios de medición con 249 indicadores, se tengan algunas brechas y por esto no están contempladas en la Resolución 0256 de 2016 y tal vez se deban incluir en el sistema de información para la evaluación de la calidad en la atención en salud del departamento de Risaralda? (24).

Todo lo anterior en el contexto de un desarrollo de modelos de anticipación que podrían modelarse con técnicas de anticipación, con programas de planificación de efectos esperados y podrían ser medidos en un periodo de tiempo con un impacto esperado adecuado, para no caer en la cotidianidad del ASIS del año pasado para implementar en este siguiente y así pensar qué va a pasar en dos años, cinco años y hasta en la siguiente década. Basados en dominios como la efectividad de la atención, la gestión del riesgo, la seguridad de la atención y la experiencia de la atención. Así, el argumento de trasformación al entrar en la modernización con el desarrollo de herramientas modernas de gestión evidencia el riesgo administrativo (17). 


\section{Referencias bibliográficas}

1. World Health Organization. Patien Safety: Marco Conceptual de la Clasificación Internacional para la Seguridad del Paciente. Informe Técnico; 2009. Disponible en https://www.who.int/patientsafety/implementation/taxonomy/ icps_technical_report_es.pdf.

2. ISO IS. International standard ISO 9000 (Quality management systems-Fundamentals and vocabulary). Vernier G, Switzerland; 2015.

3. Botero A. Calidad en salud en Colombia: Los Principios. Bogotá, D.C.; 2008. Preparado para Ministerio de Protección Social, República de Colombia.

4. Secretaría de Salud. La calidad de la atención en la salud en México a través de sus instituciones: 12 años de experiencia. Editorial: Secretaría de Salud. In: Delegación Cuauhtémoc M, D.F.; 2012.

5. República de Colombia. Ministerio de la Protección Social. Resolución 0429; 2016.

6. República de Colombia. Ministerio de la Protección Social. Informe accional de calidad de la atención en salud "INCAS Colombia 2015”, Bogotá DC; 2015.

7. OMS. International Classification for Patient Safety (ICPS). [Internet]. 2009 [citado 2017 jul. 29]. Disponible en http://www. who.int/patientsafety/implementation/ taxonomy/es/

8. OMS. Las leyes para proteger la lactancia materna son inadecuadas en la mayoría de los países. [Internet]. 2016 [citado 2017 jul. 29]. Disponible en http://www.who.int/mediacentre/news/ releases/2016/breastfeeding/es/

9. República de Colombia. Congreso de Colombia. Ley 1438 del 19 enero de
10. República de Colombia. Congreso de Colombia. Ley 1751 del 16 de febrero de 2015.

11. República de Colombia. Ministerio de Protección Social. Resolución 3253 del 3 de septiembre de 2009.

12. Ministerio de Salud y Protección Social. República de Colombia. Decreto 1011 de 2006.

13. Caballero U, Carlo V. ALM: Marketing en salud. Salud UniNorte. 2008;24(2):1-2.

14. Ministerio de Salud y Protección Social. Sistema de evaluación y calificación de actores: Ranking de satisfacción EPS. Editorial: Oficina de Calidad. In: Bogotá D.C.; 2017.

15. Ministerio de Salud y Protección Social. Incentivos al talento Humano en Salud. Editorial: Coordinación Grupo Ejercicio y Desempeño de Talento Humano en Salud, Bogotá, D.C.; 2016.

16. Moreno G. El nuevo modelo de atención integral en salud, MIAS, para Colombia: ¿La solución a los problemas del sistema? Revista médica de Risaralda. 2016;22:73-4.

17. República de Colombia. Ministerio de Salud y Protección Social. Gestión del mejoramiento continuo de la calidad MOCA, Orientaciones técnicas, gestión, resultados, monitoreo y calidad. In: Bogotá DC, Colombia, diciembre de 2016, editor. Gestión del mejoramiento continuo de la calidad; 2016.

18. República de Colombia. Ministerio de Educación. Ley 715 del 21 de diciembre de 2001.

19. Restrepo J, Rojas K. La génesis de la Economía de la Salud en Kenneth Arrow (1963). Lecturas de Economía. 2016(84):2016(84):1-34. 
20. Ossa J, Avendaño R. El pluralismo estructurado de Londoño y Frenk frente a la articulación y modulación del Sistema General de Seguridad Social en Salud (SGSSS) en Colombia. Sociedad y Economía. 2012;23:183-204.

21. República de Colombia. Ministerio de Salud y Protección Social. Resolución 518 del 24 de febrero de 2015.
22. República de Colombia. Gobernacion del Departamento de Risaralda. Secretaria de Salud. Resolución n. ${ }^{\circ} 056$ del 27 de febrero de 2009.

23. Congreso de Colombia. Ley 1797 del 13 de julio de 2016.

24. República de Colombia. Ministerio de Salud y Protección Social. Resolución 0256 del 5 de febrero de 2016. 